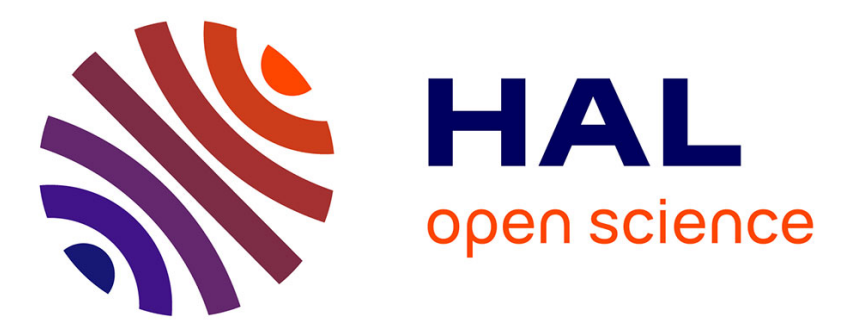

\title{
Nonparametric Identification of Nonlinear Systems in Series
}

\author{
Antonin Novak, Balbine Maillou, Pierrick Lotton, Laurent Simon
}

\section{To cite this version:}

Antonin Novak, Balbine Maillou, Pierrick Lotton, Laurent Simon. Nonparametric Identification of Nonlinear Systems in Series. IEEE Transactions on Instrumentation and Measurement, 2014, 63 (8), pp.2044-2051. 10.1109/TIM.2014.2303552 . hal-02504308

\section{HAL Id: hal-02504308 \\ https://hal.science/hal-02504308}

Submitted on 10 Mar 2020

HAL is a multi-disciplinary open access archive for the deposit and dissemination of scientific research documents, whether they are published or not. The documents may come from teaching and research institutions in France or abroad, or from public or private research centers.
L'archive ouverte pluridisciplinaire HAL, est destinée au dépôt et à la diffusion de documents scientifiques de niveau recherche, publiés ou non, émanant des établissements d'enseignement et de recherche français ou étrangers, des laboratoires publics ou privés. 


\title{
Non-parametric identification of nonlinear systems in series
}

\author{
Antonin Novak*, Balbine Maillou, Pierrick Lotton, Laurent Simon \\ Laboratoire d'Acoustique de I'Université du Mans, LAUM - UMR 6613 CNRS, Le Mans Université, Avenue Olivier \\ Messiaen, 72085 LE MANS CEDEX 9, France \\ *antonin.novak@univ-lemans.fr, *https : //ant-novak.com
}

\begin{abstract}
In this paper, a method allowing the identification of two nonlinear systems in series is presented. More precisely, the identification of the second nonlinear subsystem under test is achieved by taking into account the effects of the nonlinearities of the first subsystem. The method is based on the estimation of the Higher Harmonic Frequency Responses (HHFRs) from the measurement of distorted input and output signals. The second nonlinear system is then modeled by non-parametric generalized Hammerstein model made up of power series associated with linear filters. The method is experimentally validated in the well-known framework of nonlinear propagation of acoustic waves.
\end{abstract}

The archived file is not the final published version of the article A. Novak, B. Maillou, P. Lotton \& L. Simon (2014), "Nonparametric Identification of Nonlinear Systems in Series", Instrumentation and Measurement, IEEE Transactions on. Vol. 63(8), pp. 2044-2051.

The definitive publisher-authenticated version is available online at https://doi.org/10.1109/TIM. 2014.2303552, Readers must contact the publisher for reprint or permission to use the material in any form. 


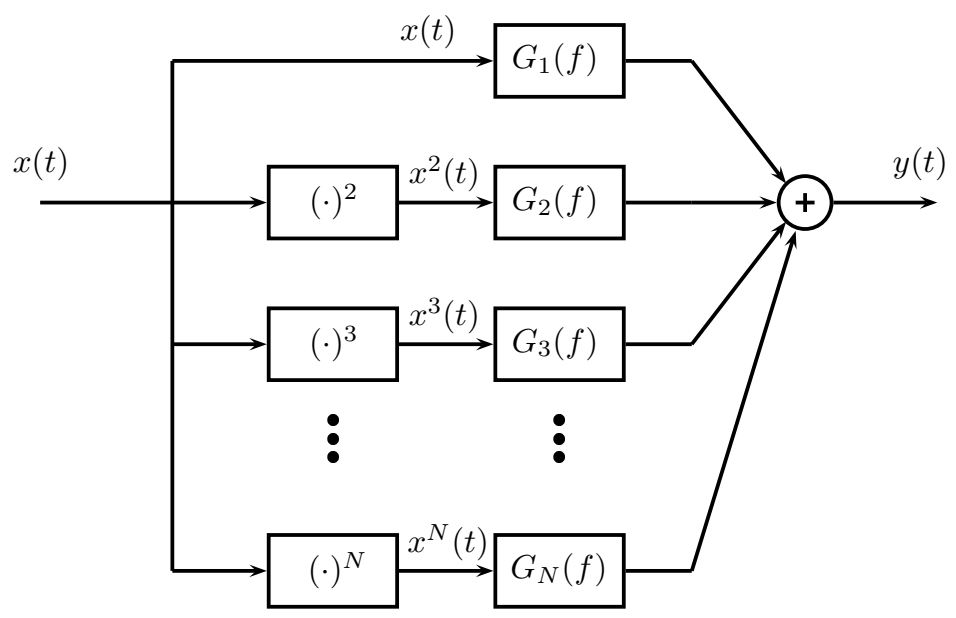

Figure 1. Generalized Hammerstein model of a SISO system.

\section{Introduction}

Almost all real-world systems exhibit nonlinear behavior to varying degrees and must be described using a nonlinear model. For such nonlinear systems, the Volterra series representation is usually considered as an effective model and its use in nonlinear system identification and analysis has become widespread from the early 80's [1], [2], [3]. Nevertheless, the Volterra model lays down the calculation of multidimensional kernels and most applications are limited to the second or third order, because of analytical difficulties and computational cost. Simplified Volterrabased models, namely Hammerstein model (N-L), Wiener model (L-N), Hammerstein-Wiener cascade (N-L-N) or Wiener-Hammerstein cascade (L-N-L) are then often preferred in the case of open-loop systems, because of their simpler structure and lower computational cost. Furthermore, for a better accuracy of the estimation, these simple models can be extended to so-called generalized models, such as the generalized Hammerstein model, as illustrated in Fig. 1, in the case of a Single-Input Single-Output (SISO) system. This generalized Hammerstein model is made up of $N$ parallel branches, with each branch consisting of a linear filter $G_{n}(f)$ preceded by a $n$th order power static nonlinear function, for $n=1, N$.

The identification of a nonlinear system using a generalized Hammerstein model consists in estimating the unknown linear filters $G_{n}(f)$ from the known input and output signals $x(t)$ and $y(t)$ respectively. Most of identification methods of Hammerstein or Wiener systems are based on parametric models [4], which means that each unknown linear filter $G_{n}(f)$ is modeled by a parametric frequency response function (FRF) model such as ARMA structure. However, a non-parametric method based on exponential swept-sine input signal $x(t)$ and nonlinear convolution has been proposed in [5] and [6] for the estimation of the modulus $\left|G_{n}(f)\right|$, and recently extended to the estimation of both the modulus and the phase of $G_{n}(f)$ through the synchronization of the excitation swept sine signal [7], [8]. This method, called Synchronized Swept-Sine Method in this paper, has been successfully applied to the study of various nonlinear systems [9], [10], [8].

However, for experimental situations, where a physical source (sound, light, vibration...) is driven by a sweptsine input signal for exciting the system under test (SUT), the unwanted nonlinearities of the source will be mixed with the nonlinearities of the SUT to be estimated. Then it might be of great interest to model the whole system (source + SUT) as two nonlinear dynamic systems in series, in order to be able to estimate independently each physical subsystem. 
The present work consequently aims at developing a method allowing the identification of the nonlinear SUT without taking into account the effects of the nonlinearities of the first subsystem. Recently, Ege et al. [11] have proposed such a method for the estimation of the nonlinearities of a piano soundboard, when the soundboard is acoustically excited with a loudspeaker. Since both the source and the soundboard are nonlinear, the characterization of the piano soundboard requires measurements of both the distorted pressure generated by the loudspeaker and the local acceleration of the soundboard. The method presented by Ege et al. then allows the estimation of the distortion rate of the soundboard only, but does not allow to identify the different contributions of the nonlinearities of the SUT.

In this paper, an off-line method allowing the identification of the second subsystem of two nonlinear systems in series is presented. This method is based on the estimation of the Higher Harmonic Frequency Responses (HHFRs) [9]. In Section 2, the principle of the method is detailed. In Section 3, the method is experimentally validated in the well-known framework of nonlinear propagation of acoustic waves. Finally, conclusions are presented in section 4.

\section{2. method}

Consider two SISO stable, time-invariant nonlinear systems (NLS) connected in series in an open loop, as depicted in Fig. 2.

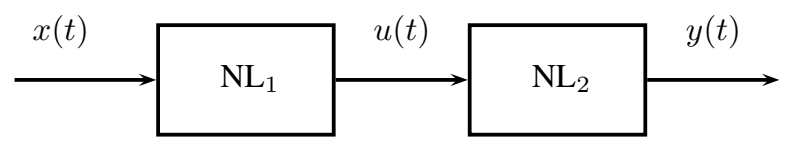

Figure 2. Two dynamic nonlinear systems in series.

The signal $x(t)$ is the input of the first NLS. The signal $u(t)$ is the output of the first NLS and the input of the second NLS. The signal $y(t)$ is the output of the second NLS. Consequently, $y(t)$ may be seen as the output of the whole system for the input signal $x(t)$. Lastly, it is supposed that these three signals are available for the analysis.

The method presented here then allows the identification of the second NLS. Adding to the assumption that both subsystems are in series without any feedback (open-loop systems), as shown in Fig. 2, we suppose in the following that the first NLS belongs to the period-in-same-period-out (PISPO) class of systems [4], and that the second NLS may be described by a $N$ th-order generalized Hammerstein model, as shown in Fig. 3.

The identification of the system is then equivalent to estimating the linear filters $G_{n}(f), n=1, N$, from the measured signals $u(t)$ and $y(t)$, the input signal $x(t)$ being known. The identification process is based on the off-line estimation of both the HHFRs $\mathcal{H}_{l}^{(u, x)}(f)$ between $x(t)$ and $u(t)$, and the HHFRs $\mathcal{H}_{l}^{(y, x)}(f)$ between $x(t)$ and $y(t)$, for $l=1, L$.

We recall that, given an input signal $a(t)$ and an output signal $b(t)$ of a NLS, the $\operatorname{HHFR} \mathcal{H}_{l}^{(b, a)}(f)$ may be seen as the contribution, in both amplitude and phase, of the $l$-th harmonic at the output, for a sine at frequency $f$ at the input, as

$$
\mathcal{H}_{l}^{(b, a)}(f)=\left|\mathcal{H}_{l}^{(b, a)}(f)\right| e^{j \varphi_{l}^{(b, a)}(f)} .
$$

Therefore, the HHFRs can be interpreted as frequency responses of fundamental and higher harmonics [9]. Several methods have been developed to estimate the HHFRs. The most intuitive but time consuming one is based on an harmonic excitation of the SUT and the process is repeated by changing the input frequency step by step. In [12], two methods have been proposed, the first one being based on FFT techniques to estimate the 


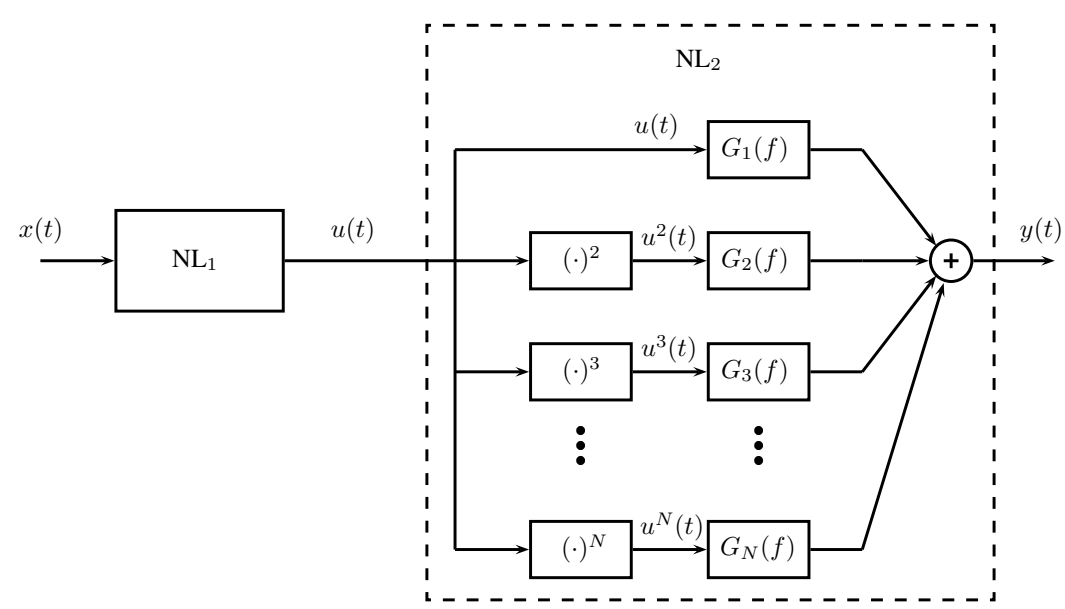

Figure 3. Two dynamic nonlinear systems in series, the second one being the system under test, represented by a generalized Hammerstein model.

auto-spectrum and phase information of HHFRs and the second one using IQ demodulation. In this paper, the so-called Synchronized Swept-Sine Method [9] is used to estimate the HHFRs, but any other technique allowing the estimation of $\mathcal{H}_{l}^{(y, x)}(f)$ and $\mathcal{H}_{l}^{(u, x)}(f)$ in both amplitude and phase can be used. The method we propose also involves estimating the HHFRs $\mathcal{H}_{l}^{\left(u^{n}, x\right)}(f)$ of powers of the signal $u(t)$, for $n=1, N$, corresponding to each polynomial input of the generalized Hammerstein model describing the second NLS. The signal $u(t)$, already distorted by the first

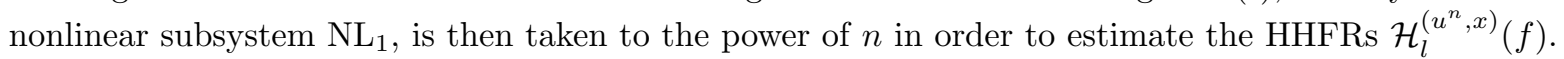

The HHFRs $\mathcal{H}_{l}^{(y, x)}(f)$ of the output signal $y(t)$ result in the combination of all HHFRs $\mathcal{H}_{l}^{\left(u^{n}, x\right)}(f)$ after filtering by filters $G_{n}(f)$. As detailed in Appendix A.1, the relation between the HHFRs $\mathcal{H}_{l}^{\left(u^{n}, x\right)}(f), \mathcal{H}_{l}^{(y, x)}(f)$ and the linear filters $G_{n}(f)$ can indeed be written in a matrix form as

$$
\begin{aligned}
{\left[\begin{array}{c}
\mathcal{H}_{1}^{(y, x)}(f) \\
\mathcal{H}_{2}^{(y, x)}(f) \\
\vdots \\
\mathcal{H}_{L}^{(y, x)}(f)
\end{array}\right]=} & {\left[\begin{array}{cccc}
\mathcal{H}_{1}^{(u, x)}(f) & \mathcal{H}_{1}^{\left(u^{2}, x\right)}(f) & \cdots & \mathcal{H}_{1}^{\left(u^{N}, x\right)}(f) \\
\mathcal{H}_{2}^{(u, x)}(f) & \mathcal{H}_{2}^{\left(u^{2}, x\right)}(f) & \cdots & \mathcal{H}_{2}^{\left(u^{N}, x\right)}(f) \\
\vdots & \vdots & \ddots & \vdots \\
\mathcal{H}_{L}^{(u, x)}(f) & \mathcal{H}_{L}^{\left(u^{2}, x\right)}(f) & \ldots & \mathcal{H}_{L}^{\left(u^{N}, x\right)}(f)
\end{array}\right] } \\
& \times\left[\begin{array}{c}
G_{1}(f) \\
G_{2}(f) \\
\vdots \\
G_{N}(f)
\end{array}\right] .
\end{aligned}
$$

Equation (2) can then be solved for unknown $G_{n}(f)$ by using a square matrix inversion in the case $L=N$, or by using a pseudo-inversion in the case $L>N$. The number of harmonics $L$ chosen for the estimation of HHFRs must be equal or greater than the number of the branches $N$ of the generalized Hammerstein model. The matrix (pseudo)-inversion must be computed for each frequency $f$ separately. Nevertheless, for frequencies corresponding to weak values of the output $u(t)$ of the first nonlinear system, the matrix $L \times N$ may be ill-conditioned. This can lead to meaningless solutions. Consequently, the values of $\mathcal{H}_{l}^{(u, x)}(f)$ have to be calculated first in order to achieve a good matrix conditioning. Appendix A.1 details the method when exciting the two nonlinear systems in series by a sine wave of frequency $f_{0}$. 


\section{Validation on an Acoustic Waveguide}

\subsection{Experimental set up description}

To illustrate and validate the method described in the previous section, a particular case of two nonlinear systems connected in series is considered here. It consists of a compression driver exciting an acoustic waveguide as shown in Fig. 4.

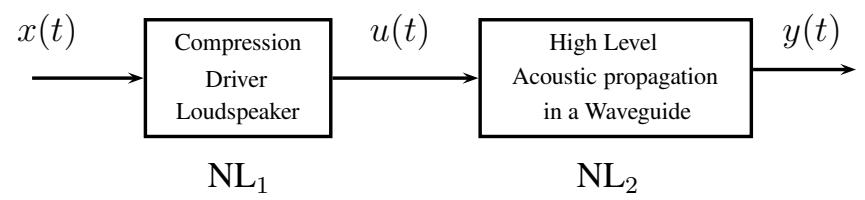

Figure 4. Two real-world dynamic nonlinear systems in series.

A high level excitation voltage is supplied to the driver so that the behavior of the driver is supposed to be nonlinear. The driver generates a high level acoustic signal at the input of the waveguide so that a nonlinear acoustic propagation occurs in the waveguide. Nonlinear acoustic propagation due to a high level source can lead to shock wave for long distance propagation [13]. Indeed, local small nonlinear perturbations of the acoustic wave due to high sound pressure level are cumulative along the propagation and may distort the waveform considerably for sufficiently large source-to-receiver distances. Nevertheless, interest is focused here on a shorter distance, leading to the observation of weak nonlinear distortion, mathematically described by Burgers' equation [14], as summarized in Appendix B.

In addition to this weak nonlinear distortion, the acoustic pressure generated at the input of the waveguide is distorted, due to the nonlinear behavior of the driver, in such a way that the acoustic pressure propagating in the waveguide contains additional loudspeaker-driven nonlinear components. Identification of the nonlinearities solely due to nonlinear propagation in the waveguide is then carried out, using the identification method presented in the previous section. The experimental setup is depicted in Fig.5.

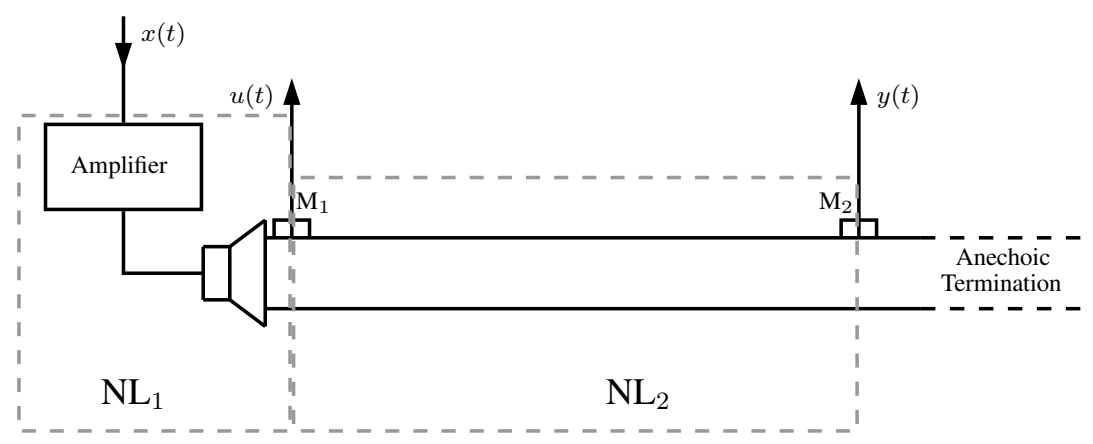

Figure 5. Schematic representation of the experimental setup.

The setup is made up of a cylindrical air-filled tube $(6 \mathrm{~m}$ long and $58 \mathrm{~mm}$ internal diameter) coupled to a compression driver (JBL model $2446 \mathrm{H}$ ) at one of its ends. The other end of the tube is loaded by an absorbing termination in order to avoid sound reflections. The input signal $x(t)$ is a swept sine satisfying the conditions required for the Synchronized Swept-Sine Method, as detailed in [7]. Two microphones M1 and M2 (acceleration compensated piezo-electrical gauges PCB M116B) are flush mounted on the pipe wall. The first one is set at $20 \mathrm{~cm}$ from the source, whereas the second one is set at the distance of $4.2 \mathrm{~m}$ from the source. In this experiment, the first NLS consists of the power amplifier, the compression driver and the part of the guide between the driver and 
the first microphone. The second NLS is the part of the guide between the two microphones.

Pressure level provided by the driver at the input of the tube is chosen to be high enough for exhibiting weakly nonlinear acoustic propagation. In this experiment, the RMS pressure is $650 \mathrm{~Pa}$. Measurements are performed from $300 \mathrm{~Hz}$ to $5000 \mathrm{~Hz}$ and sampling frequency is set to $96 \mathrm{kHz}$. For this frequency range, the absorbing termination is efficient, so that the outgoing wave reflected from the end of the tube is negligible. Thus, the identification results should be compared with the nonlinear traveling plane wave theory, based on Burgers' equation resolution and detailed in Appendix A.2. Note that the cutoff frequency, below which the plane wave approximation is valid, is around $3300 \mathrm{~Hz}$.

\subsection{Estimation of Higher Harmonic Frequency Responses}

Both acoustic pressure signals $u(t)$ and $y(t)$ (from microphones M1 and M2) are independently analyzed thanks to the Synchronized Swept-Sine Method, by estimating HHFRs up to the 9th-order HHFR, with $x(t)$ the input signal. In the following, only the first, second and third HHFRs are plotted to improve readability of the graphs. In Fig. 6, the moduli of the HHFRs $\mathcal{H}_{l}^{(u, x)}(f)$ at the first microphone location are given. Similarly, Fig. 7 shows the moduli of the HHFRs $\mathcal{H}_{l}^{(y, x)}(f)$ at the second microphone location.

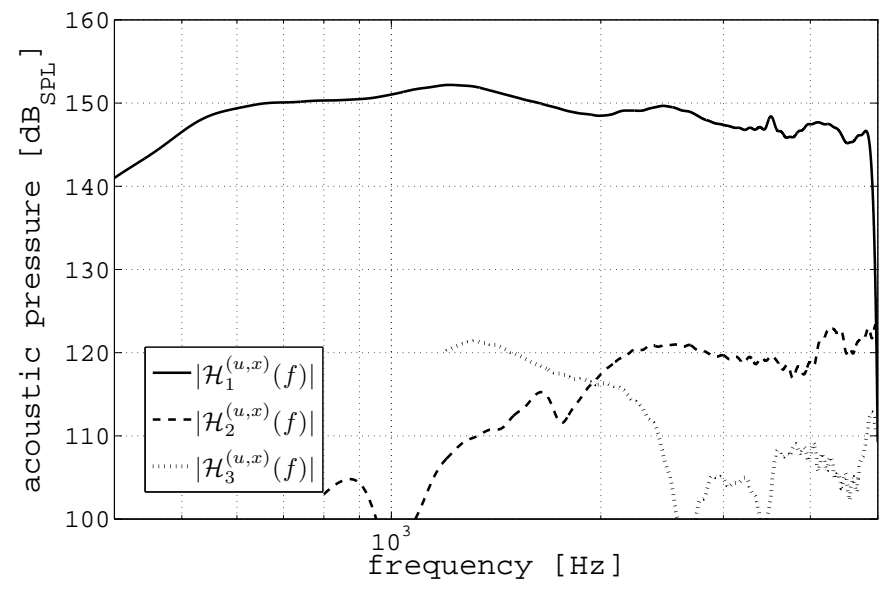

Figure 6. HHFRs $\left|\mathcal{H}_{l}^{(u, x)}(f)\right|$ at the first microphone location. Only the first, second and third HHFRs are plotted.

Fig. 6 and 7 may be read as follows. For a sine wave excitation at a given frequency $f$, the acoustic pressure signal under analysis is made up of a fundamental frequency, a second and a third-order frequency components, which amplitude level is available at $f, 2 f$ and $3 f$ frequencies, respectively.

Such representation of $l$ th-order harmonics allows to read easily the levels of the HHFRs, relatively to a given value of the fundamental and consequently to evaluate the distortion, for each input frequency.

In Fig. 6, fundamental level of first microphone acoustic pressure signal, $\left|\mathcal{H}_{1}^{(u, x)}(f)\right|$, is quite constant, around 140-150 dBSPL over the whole frequency span. The level of second harmonic $\left|\mathcal{H}_{2}^{(u, x)}(f)\right|$ is lower than the fundamental one and increases slightly with frequency. $\left|\mathcal{H}_{3}^{(u, x)}(f)\right|$ level is lower than $\left|\mathcal{H}_{2}^{(u, x)}(f)\right|$ except below $800 \mathrm{~Hz}$ input frequency, leading to a minimum dynamic value between $\left|\mathcal{H}_{1}^{(u, x)}(f)\right|$ and $\left|\mathcal{H}_{3}^{(u, x)}(f)\right|$ of $25 \mathrm{~dB}$, for an input frequency of $450 \mathrm{~Hz}$.

Comparing the results at both microphones locations, the level of $\left|\mathcal{H}_{1}^{(y, x)}(f)\right|$ measured by the second microphone is $1 \mathrm{~dB}$ lower than the level of $\left|\mathcal{H}_{1}^{(u, x)}(f)\right|$ measured by the first microphone, for low frequencies. Moreover, the difference between fundamental levels at both microphones locations increases slightly with frequency above $2000 \mathrm{~Hz}$. Both these effects are due to visco-thermal losses, which theoretically increase with frequency [15]. Furthermore, 


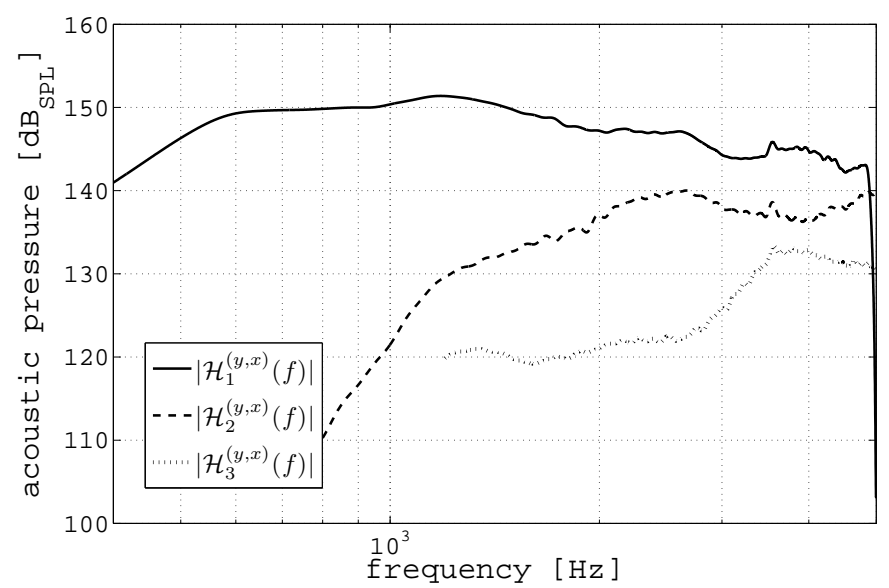

Figure 7. HHFRs $\left|\mathcal{H}_{l}^{(y, x)}(f)\right|$ at the second microphone location. Only the first, second and third HHFRs are plotted.

we notice in Fig. 7 that the acoustic pressure at the second microphone location is more distorted than at the first microphone location. This is due to the effects of nonlinear propagation: $\left|\mathcal{H}_{2}^{(y, x)}(f)\right|$ and $\left|\mathcal{H}_{3}^{(y, x)}(f)\right|$ globally increase with frequency, as predicted by the theory [16].

\subsection{Generalized Hammerstein Model and Burgers' Theory}

From experimental signals $x(t), u(t)$ and $y(t)$, HHFRs $\mathcal{H}_{l}^{\left(u^{n}, x\right)}(f)$ and $\mathcal{H}_{l}^{(y, x)}(f)$ are calculated, with $l=1,9$. Then, the linear filters $G_{n}(f)$, for $n=1,3$, are estimated from (2). They characterize the behavior of the second nonlinear system, as far as a generalized Hammerstein model correctly fits the nonlinear propagation in the waveguide. The HHFRs $\mathcal{H}_{l}^{(y, u)}(f)$ for a given input $u(t)$ may then be calculated from the identified generalized Hammerstein model as depicted in Fig. 8.

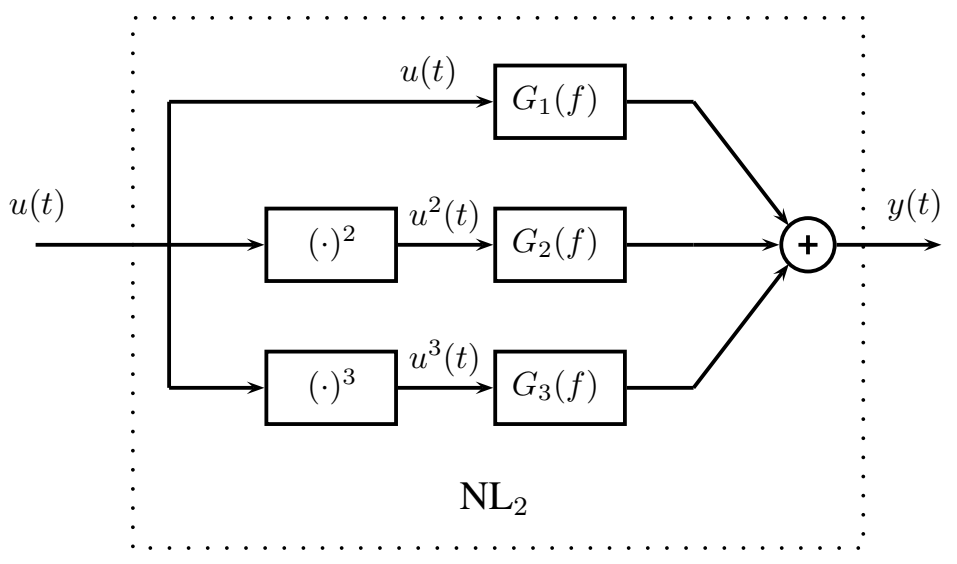

Figure 8. Generalized Hammerstein model of the nonlinear propagation in the waveguide. Linear filters $G_{n}(f)$ are obtained from experimental data.

Moreover, the nonlinear acoustic propagation in the waveguide is completely described by the theory of Burgers detailed in Appendix A.2. A theoretical expression of HHFRs for a given input pressure is then available, noted $H_{l}(f)$.

As a validation, we aim at comparing the calculated HHFRs $\mathcal{H}_{l}^{(y, u)}(f)$ from the estimated model and the theoretical HHFRs $H_{l}(f)$. Besides, we also compare in the following the waveforms of the measured, theoretical 
and model-based re-synthesized acoustical pressures.

Two comparisons are presented here. First, in Figs. 9 and 10, the theoretical results calculated using Burger's theory are compared with the measured data including the whole system $\left(\mathrm{NL}_{1}\right.$ in series with $\left.\mathrm{NL}_{2}\right)$. This comparison represents a case for which the nonlinearities of the first subsystem $\left(\mathrm{NL}_{1}\right)$ are mixed with the nonlinearities of the $\operatorname{SUT}\left(\mathrm{NL}_{2}\right)$.

In Fig. 9, the theoretical HHFRs $H_{l}(f)$ are compared with the HHFRs $\mathcal{H}_{l}^{(y, x)}(f)$ of the whole system which contains information of nonlinearities of both systems $\mathrm{NL}_{1}$ and $\mathrm{NL}_{2}$. Discrepancies between theoretical $H_{l}(f)$ and measured $\mathcal{H}_{l}^{(y, x)}(f)$ are then obvious, especially for second and third HHFRs. In particular, $\mathcal{H}_{3}^{(y, x)}(f)$ overestimates $H_{3}(f)$ in the frequency span from $300 \mathrm{~Hz}$ up to $800 \mathrm{~Hz}$, due to the compression driver distortion contribution, as noted in Fig. 6.

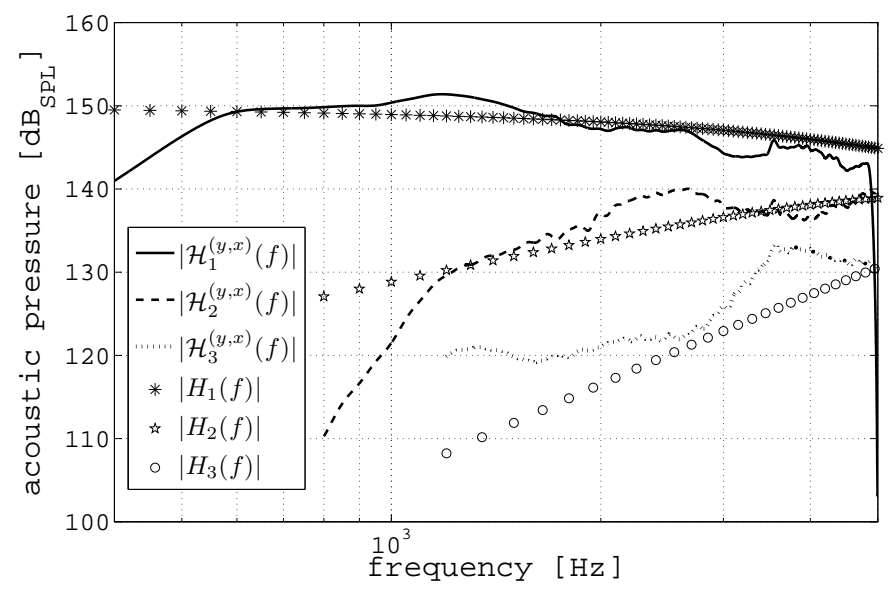

Figure 9. Comparison between the theoretical HHFRs $H_{l}(f)$ and the estimated $\mathcal{H}_{l}^{(y, x)}(f)$. The nonlinearities of the first subsystem $\left(\mathrm{NL}_{1}\right)$ are mixed with the nonlinearities of the SUT $\left(\mathrm{NL}_{2}\right)$.

Fig. 10 shows the comparison of the time waveforms of the acoustical pressure for input signal at $1400 \mathrm{~Hz}$. The theoretical waveform calculated using the Burger's theory (dashed line) clearly differs from the experimental signal captured by the microphone 2 (solid line), the RMS error between both waveforms reaching $54.3 \mathrm{~Pa}$ due to the nonlinearities of the first subsystem $\left(\mathrm{NL}_{1}\right)$.

The second comparison (Figs. 11 and 12) shows the theoretical results calculated using Burger's theory compared with the estimated model. In other words, the identification of the second nonlinear subsystem (the acoustical waveguide) is achieved while getting rid of the effects of the first subsystem (the compression driver), according to Equation (2).

In Fig. 11, the theoretical HHFRs $H_{l}(f)$ are compared with the HHFRs $\mathcal{H}_{l}^{(y, u)}(f)$ obtained from the estimated model of the SUT $\left(\mathrm{NL}_{2}\right)$. The results show that the identification of the propagation in the waveguide $\left(\mathrm{NL}_{2}\right)$ is in agreement with Burgers' theory, contrary to the results proposed in Fig. 9.

The same conclusion can be drawn with regard to the comparison of time waveforms of the acoustical pressure (Fig. 12). The waveform (solid line) re-synthesized from the estimated model matches well with the expected theoretical waveform calculated using the Burger's theory (dashed line), the RMS error being 9.3 Pa, almost six times lower than in the previous case.

The accuracy of the proposed identification is also confirmed by calculating the root mean squared error $E_{l}$ for 


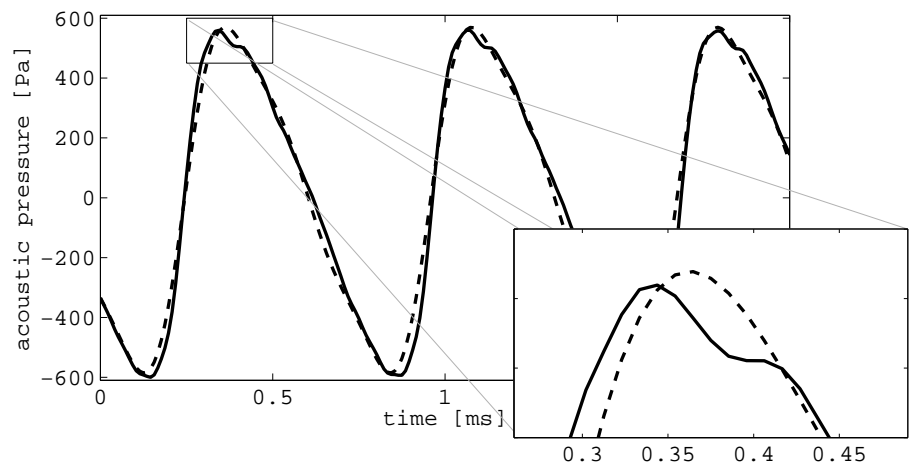

Figure 10. Time waveforms of the acoustical pressure captured by microphone 2 for an input signal at $1400 \mathrm{~Hz}-$ solid line; the theoretical time waveform calculated using the Burger's theory - dashed line.

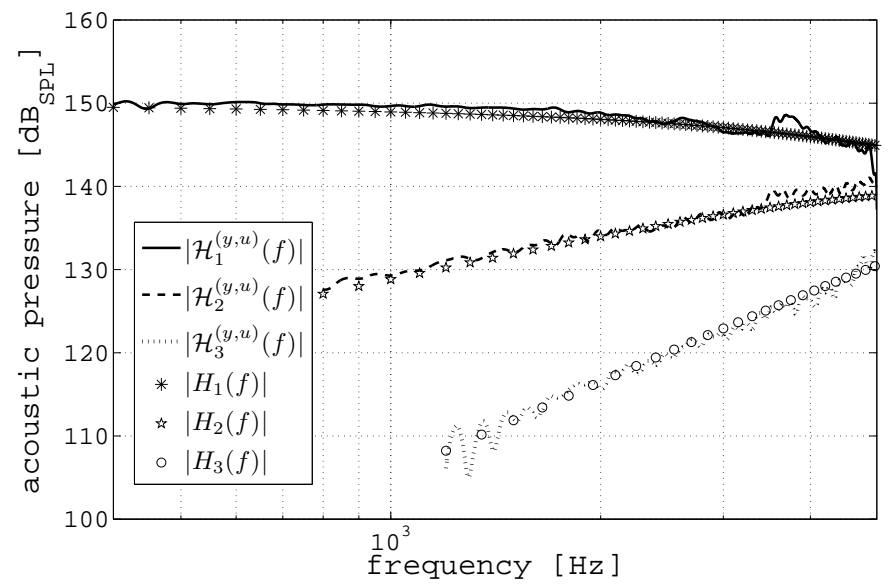

Figure 11. Comparison between the theoretical HHFRs $H_{l}(f)$ and the calculated $\mathcal{H}_{l}^{(y, u)}(f)$. The SUT $\left(\mathrm{NL}_{2}\right)$ is estimated independently of the nonlinearities of the first subsystem $\mathrm{NL}_{1}$.

each HHFR $\mathcal{H}_{l}^{(y, u)}(f)$ as

$$
E_{l}=\frac{\sqrt{\frac{1}{N} \sum_{k=1}^{N}\left(\left|H_{l}\left(f_{k}\right)\right|-\left|\mathcal{H}_{l}^{(y, u)}\left(f_{k}\right)\right|\right)^{2}}}{\sqrt{\frac{1}{N} \sum_{k=1}^{N}\left(\left|H_{l}\left(f_{k}\right)\right|\right)^{2}}}
$$

for which values are given, for both cases, in Table 1.

\section{Conclusion}

In this paper, a method for the identification of two NLS in series has been presented.

The cascade of two NLS means that the second NLS under test is excited by a distorted signal, which is the output of the first nonlinear subsystem. In practice, this corresponds to the classical identification case of a system under test excited by a nonlinear device, as a loudspeaker or a shaker.

The method proposed in this paper identifies the second system using a generalized Hammerstein representation. On one hand, one of the characteristics of the method is to properly estimate the HHFRs of input and output of nonlinear SUT. Among several available methods, the Synchronized Swept-Sine method has been chosen for both its rapidity and robustness [7]. 


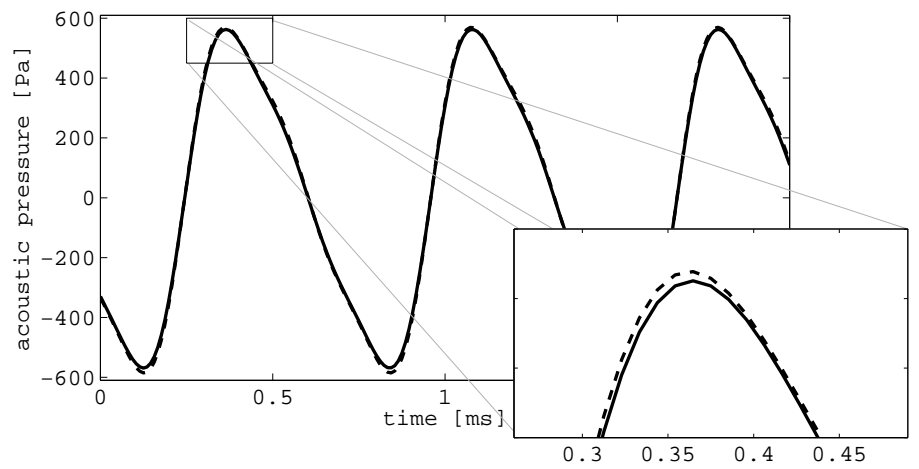

Figure 12. Time waveforms of the acoustical pressure for an input signal at $1400 \mathrm{~Hz}$ : re-synthesized waveform from the estimated model - solid line; the theoretical waveform calculated using the Burger's theory - dashed line.

\begin{tabular}{c|c|c} 
& First case & Second case \\
\hline 1st HHFR & 0.21 & 0.08 \\
\hline 2nd HHFR & 0.40 & 0.05 \\
\hline 3rd HHFR & 0.6 & 0.10
\end{tabular}

Table 1. Normalized root mean squared error $E_{l}$, for first and second cases, respectively corresponding to Fig. 9 and 11 .

On the other hand, it is also worth noting that the method is low time consuming. The proposed method is indeed easy to implement and no special algorithm is required. In addition, the method has no need for any knowledge of the first subsystem, as far as the generalized Hammerstein model correctly fits the nonlinear behavior of the (second) nonlinear system under test.

The method allows in particular to regenerate an output signal corresponding to any given input signal. Thanks to this property we validate the method for a theoretically very well-known physical system, i.e. the weakly nonlinear acoustic propagation in a waveguide.

The results show good agreement between both model-based and theoretical system outputs.

\section{A.1 Detailed procedure}

The following example details the estimation procedure of the unknown filters $G_{n}(f)$ when exciting the cascade of nonlinear systems by a sine wave signal. In this example, the nonlinear system under test (the second nonlinear system) is represented by a third order generalized Hammerstein model. The estimation procedure is detailed for a frequency $f_{0}$, leading to estimation of the three unknowns $G_{1}\left(f_{0}\right), G_{2}\left(f_{0}\right)$ and $G_{3}\left(f_{0}\right)$.

First, we excite the cascade of nonlinear systems with a sine wave signal $x(t)$ with frequency $f_{0}$, as depicted in Fig. 13. For frequency $f_{0}$, the relation between the input $u(t)$ and the output $y(t)$ of the nonlinear system under test can be written as

$$
\begin{aligned}
& \mathcal{H}_{1}^{(u, x)}\left(f_{0}\right) \cdot G_{1}\left(f_{0}\right)+\mathcal{H}_{1}^{\left(u^{2}, x\right)}\left(f_{0}\right) \cdot G_{2}\left(f_{0}\right) \\
& +\mathcal{H}_{1}^{\left(u^{3}, x\right)}\left(f_{0}\right) \cdot G_{3}\left(f_{0}\right)=\mathcal{H}_{1}^{(y, x)}\left(f_{0}\right) .
\end{aligned}
$$

This equation describes the relation between the first harmonics of signals $u(t), u^{2}(t), u^{3}(t)$ and $y(t)$. 
Next, we change the excitation frequency of the excitation signal $x(t)$ to $\frac{f_{0}}{2}$ and we study what happens for the same frequency $f_{0}$ as in the previous case. This situation is illustrated in Fig. 14 and leads to an equation that describes the relation between the second harmonics of signals $u(t), u^{2}(t), u^{3}(t)$ and $y(t)$, as

$$
\begin{aligned}
& \mathcal{H}_{2}^{(u, x)}\left(f_{0}\right) \cdot G_{1}\left(f_{0}\right)+\mathcal{H}_{2}^{\left(u^{2}, x\right)}\left(f_{0}\right) \cdot G_{2}\left(f_{0}\right) \\
& +\mathcal{H}_{2}^{\left(u^{3}, x\right)}\left(f_{0}\right) \cdot G_{3}\left(f_{0}\right)=\mathcal{H}_{2}^{(y, x)}\left(f_{0}\right) .
\end{aligned}
$$

Finally, we change the excitation frequency of the excitation signal $x(t)$ to $\frac{f_{0}}{3}$ and we still study what happens for frequency $f_{0}$. This situation is illustrated in Fig. 15 and leads to an equation that describes the relation between the third harmonics of signals $u(t), u^{2}(t), u^{3}(t)$ and $y(t)$, as

$$
\begin{aligned}
& \mathcal{H}_{3}^{(u, x)}\left(f_{0}\right) \cdot G_{1}\left(f_{0}\right)+\mathcal{H}_{3}^{\left(u^{2}, x\right)}\left(f_{0}\right) \cdot G_{2}\left(f_{0}\right) \\
& +\mathcal{H}_{3}^{\left(u^{3}, x\right)}\left(f_{0}\right) \cdot G_{3}\left(f_{0}\right)=\mathcal{H}_{3}^{(y, x)}\left(f_{0}\right) .
\end{aligned}
$$

The systems of three equations Eqs.(A.1-A.3) in three unknowns $G_{1}\left(f_{0}\right), G_{2}\left(f_{0}\right)$ and $G_{3}\left(f_{0}\right)$ can be solved. Generalizing the number of unknowns (number of branches of the generalized Hammerstein model) to $N$ and the number of harmonics to $L$, and repeating the procedure for all the desired frequencies $f$ leads to the matrix expression (2).

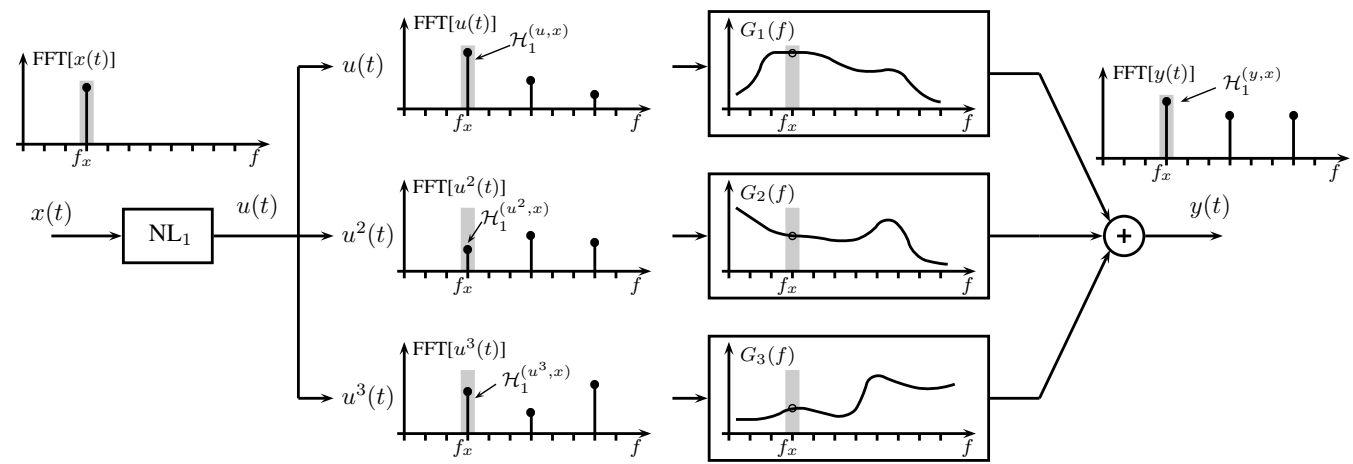

Figure 13. Outline of the proposed method. The excitation frequency of the sine wave is $f_{0}$.

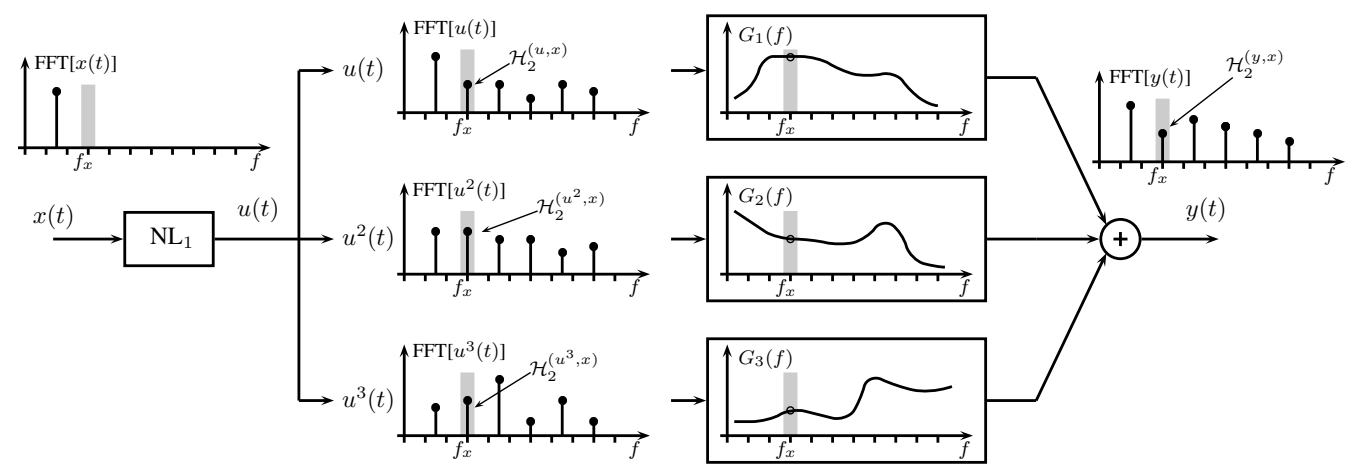

Figure 14. Outline of the proposed method. The excitation frequency of the sine wave is $\frac{f_{0}}{2}$. 


\section{A.2 Burgers' equation for traveling plane waves}

Generalized Burgers' equation with thermoviscous losses in the boundary layer, for a pure traveling wave is [16] [17] $[18]$

$$
\frac{\partial q^{+}}{\partial \sigma}=q^{+} \frac{\partial q^{+}}{\partial \theta^{+}}-\frac{T}{\epsilon} \frac{\partial q^{+}}{\partial \theta^{+}} * \frac{1}{\sqrt{\pi \theta^{+}}}
$$

with $\sigma=(\gamma+1) M \omega x /\left(2 c_{0}\right), \epsilon=(\gamma+1) M / 2, T=S h\left(1+(\gamma-1) / \sqrt{P r}, *\right.$ being the convolution, $q^{+}$the progressive acoustic pressure or velocity, $M$ the acoustic Mach number, $\gamma$ the heat capacity ration, $S h$ the shear number, $\mathrm{Pr}$ the Prandtl number, $c_{0}$ the small-signal speed of sound [16] [18], $\omega$ the angular frequency and $\theta^{+}=\omega\left(t-x / c_{0}\right)$, with $t$ and $x$ the time and space coordinates, respectively.

Equation (A.4) is built from an adapted method for cumulative phenomena, the Multiple Scale Method (MSM), which has been validated up to a distance close to the critical shock wave distance [14]. This equation has no analytical known solution.

Solution is therefore obtained numerically in the frequency domain using a Fourier series decomposition [17], expressed as

$$
q=\sum_{l=1}^{\infty}\left(a_{l}(\sigma) \sin (l \theta)+b_{l}(\sigma) \cos (l \theta)\right)
$$

Introducing (A.4) into (A.5) leads to the following expressions

$$
\begin{aligned}
\frac{\partial a_{l}}{\partial \sigma}= & l \sum_{p=1}^{l-1}\left(\frac{a_{p} a_{l-p}}{2}-\frac{b_{p} b_{l-p}}{2}\right) \\
& -l \sum_{p=l+1}^{+\infty}\left(a_{p-l} a_{p}+b_{p-l} b_{p}\right) \\
& -\frac{T}{\epsilon} \sqrt{\frac{l}{2}}\left(a_{l} b_{l}\right)
\end{aligned}
$$

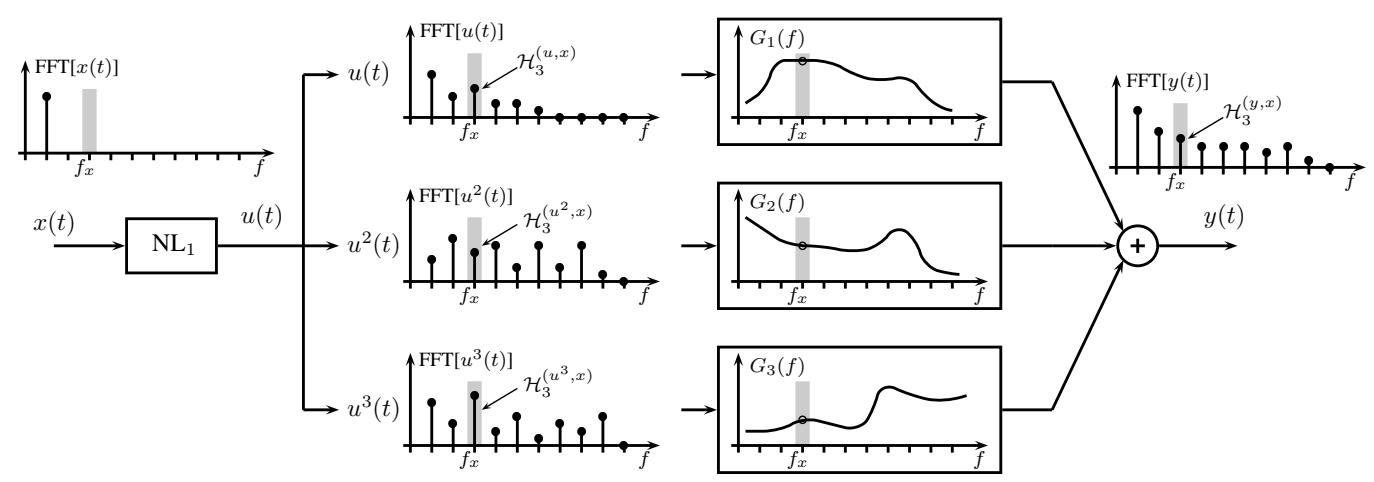

Figure 15. Outline of the proposed method. The excitation frequency of the sine wave is $\frac{f_{0}}{3}$. 


$$
\begin{aligned}
\frac{\partial b_{l}}{\partial \sigma}= & l \sum_{p=1}^{l-1}\left(\frac{a_{p} b_{l-p}}{2}-\frac{b_{p} a_{l-p}}{2}\right) \\
& -l \sum_{p=l+1}^{+\infty}\left(b_{p-l} a_{p}+a_{p-l} b_{p}\right) \\
& -\frac{T}{\epsilon} \sqrt{\frac{l}{2}}\left(a_{l}+b_{l}\right)
\end{aligned}
$$

In the frame of this work, these Fourier series are truncated to $\mathrm{L}$ harmonics, $l=1,3$. Then a resolution on $\sigma$ by small steps $\Delta \sigma$ (finite difference method) knowing the initial condition at $\sigma=0$ is performed. Spatial step is typically here $1 \mathrm{~cm}$. The resolution method used is a prediction correction method. At first order, the classical Euler method is used, and correction is performed using the Adams Moulton second order method.

The solution of Burgers' equation was experimentally verified by Menguy et al. [17]. This theory serves in this work as a reference for testing the proposed identification method, by deducing the theoretical moduli of HHFRs, according to

$$
H_{l}=\sqrt{a_{l}^{2}(\sigma)+b_{l}^{2}(\sigma)}
$$

\section{Acknowledgment}

The authors would like to thank Joel Gilbert and Michal Bednarik for their help in Burgers' theory for the numerical simulation of the HHFRs.

\section{References}

[1] M. Schetzen, The Volterra and Wiener Theories of Nonlinear Systems. New York: John Wiley \& Sons, Apr. 1980.

[2] S. Billings, "Identification of nonlinear systems : a survey," Control Theory and Applications, IEE Proceedings $D$, vol. 127 , no. 6, pp. $272-285$, Nov. 1980.

[3] T. Koh and E. Powers, "Second-order volterra filtering and its application to nonlinear system identification," IEEE Transactions on Acoustics, Speech and Signal Processing, vol. 33, no. 6, pp. 1445 - 1455, Dec. 1985.

[4] M. Schoukens, R. Pintelon, and Y. Rolain, "Parametric identification of parallel hammerstein systems," IEEE Transactions on Instrumentation and Measurement, vol. 60, no. 12, pp. 3931 -3938, 2011.

[5] A. Farina, "Simultaneous measurement of impulse response and distortion with a swept-sine technique," in Proceedings of the 108th Convention of the Audio Engineering Society, Paris, France, Feb. 2000.

[6] E. Armelloni, A. Bellini, and A. Farina, "Non-linear convolution : a new approach for the auralization of distorting systems," in Proceedings of the 110th Convention of the Audio Engineering Society, Amsterdam, The Nederlands, May 2001.

[7] A. Novak, L. Simon, F. Kadlec, and P. Lotton, "Nonlinear system identification using exponential swept-sine signal," IEEE Transactions on Instrumentation and Measurement, vol. 59, no. 8, pp. 2220 -2229, Aug. 2010.

[8] M. Rébillat and X. Boutillon, "Measurement of relevant elastic and damping material properties in sandwich thick plates," Journal of Sound and Vibration, vol. 330, no. 25, pp. 6098-6121, Dec. 2011. 
[9] A. Novak, L. Simon, and P. Lotton, "Analysis, synthesis, and classification of nonlinear systems using synchronized swept-sine method for audio effects," EURASIP J. Adv. Signal Process, vol. 2010, pp. 1-8, Feb. 2010.

[10] A. Novak, M. Bentahar, V. Tournat, R. El Guerjouma, and L. Simon, "Strutural health monitoring based on nonlinear system identification," in 10eme Congres Francais d'Acoustique, S. F. d. SFA, Ed., Lyon, France, Apr. 2010.

[11] K. Ege, X. Boutillon, and M. Rébillat, "Vibroacoustics of the piano soundboard: (non)linearity and modal properties in the low- and mid-frequency ranges," Journal of Sound and Vibration, vol. 332, no. 5, pp. 12881305, Mar. 2013.

[12] P. Nuij, O. Bosgra, and M. Steinbuch, "Higher-order sinusoidal input describing functions for the analysis of non-linear systems with harmonic responses," Mechanical Systems and Signal Processing, vol. 20, no. 8, pp. 1883-1904, Nov. 2006.

[13] A. Hirschberg, J. Gilbert, R. Msallam, and A. P. J. Wijnands, "Shock waves in trombones," The Journal of the Acoustical Society of America, vol. 99, no. 3, pp. 1754-1758, Mar. 1996.

[14] J. Burgers, "A mathematical model illustrating the theory of turbulence," in Advances in Applied Mechanics, Richard Von Mises and Theodore Von Kármán, Ed. Elsevier, 1948, vol. Volume 1, pp. 171-199.

[15] A. D. Pierce, Acoustics: An Introduction to Its Physical Principles and Applications. Acoustical Soc of America, 1989.

[16] D. T. Blackstock, "Generalized burgers equation for plane waves," The Journal of the Acoustical Society of America, vol. 77, no. 6, pp. 2050-2053, 1985.

[17] L. Menguy and J. Gilbert, "Weakly nonlinear gas oscillations in air-filled tubes; solutions and experiments," Acta Acustica united with Acustica, vol. 86, no. 5, pp. 798-810, Aug. 2000.

[18] M. Bednarik and P. Konicek, "Propagation of quasiplane nonlinear waves in tubes and the approximate solutions of the generalized burgers equation," The Journal of the Acoustical Society of America, vol. 112, no. 1, pp. 91-98, Jul. 2002. 